\title{
High Glucose Disrupts Mitochondrial Morphology in Retinal Endothelial Cells
}

\author{
Implications for Diabetic Retinopathy
}

\author{
Kyle Trudeau, ${ }^{*}$ Anthony J.A. Molina, ${ }^{*}$ Wen Guo, ${ }^{*}$ \\ and Sayon Roy* \\ From the Departments of Medicine, ${ }^{*}$ and Ophthalmology, ${ }^{\dagger}$ Boston \\ University School of Medicine, Massachusetts
}

Mitochondrial dysfunction has been implicated in diabetic complications; however, it is unknown whether hyperglycemia affects mitochondrial morphology and metabolic capacity during development of diabetic retinopathy. We investigated high glucose (HG) effects on mitochondrial morphology, membrane potential heterogeneity, cellular oxygen consumption, extracellular acidification, cytochrome c release, and apoptosis in retinal endothelial cells. Rat retinal endothelial cells grown in normal $(5 \mathrm{mmol} / \mathrm{L})$ or $\mathrm{HG}(30 \mathrm{mmol} / \mathrm{L})$ medium and double-stained with MitoTracker Green and tetramethylrhodamine-ethylester-perchlorate were examined live with confocal microscopy. Images were analyzed for mitochondrial shape change using Form Factor and Aspect Ratio values, and membrane potential heterogeneity, using deviation of fluorescence intensity values. Rat retinal endothelial cells grown in normal or HG medium were analyzed for transient changes in oxygen consumption and extracellular acidification using an XF-24 flux analyzer, cytochrome $\mathrm{c}$ release by Western blot, and apoptosis by terminal deoxynucleotidyl transferase-mediated dUTP nick-end labeling assay. Rat retinal endothelial cells grown in HG medium exhibited increased mitochondrial fragmentation concurrent with membrane potential heterogeneity. Metabolic analysis showed increased extracellular acidification in HG with reduced steady state/maximal oxygen consumption. Cytochrome $c$ and terminal deoxynucleotidyl transferase-mediated dUTP nick-end labeling-positive cells were also increased in HG. Thus, HG-induced mitochondrial fragmentation with concomitant increase in membrane potential heterogeneity, reduced oxygen consumption, and cytochrome c release may underlie apoptosis of retinal endothelial cells as seen in diabetic retinopathy. (Am J Pathol 2010, 177:447-455; DOI: 10.2353/ajpath.2010.091029)

Diabetes is characterized by hyperglycemia and consequent functional failure of various target organs including the eye. In the working-age population, diabetic retinopathy is the leading cause of blindness, ${ }^{1}$ which is triggered at least in part by hyperglycemia-induced apoptosis. While biochemical studies have implicated mitochondrial dysfunction as an underlying mechanism for inducing apoptosis, ${ }^{2}$ the implications of mitochondrial structural changes in this process have only recently begun to be examined. In most cell types, mitochondria exist as long tubular networks that are precisely regulated by the rates of mitochondrial fusion and fission events. Disruption in this delicate balance induces altered mitochondrial membrane potential heterogeneity, ${ }^{3-5}$ mitochondrial fragmentation, and apoptosis. ${ }^{6-8}$

Although oxidative stress is known to increase in diabetic retinas and trigger pro-apoptotic actions of mitochondria including the release of cytochrome $\mathrm{c}$, it is currently unclear if compromised mitochondrial structure is a necessary event for high glucose (HG)-mediated apoptosis. We have shown that $H G$ induces apoptosis in the rat retinal endothelial cells (RRECs) ${ }^{9}$ and recent studies have indicated that HG causes mitochondrial dysfunction through oxidative damage of mitochondrial DNA and contributes to apoptosis in the human retinal endothelial cells. ${ }^{10}$ In various cell types, including rat hepato-

Supported by National Eye Institute, National Institute of Health EY018218 (S.R), National Institute of Diabetes and Digestive and Kidney Diseases 5T32DK007201 (A.J.A.M.), DK59261 (W.G). Fight for Sight (K.T.), ADATakeda Pharmaceuticals Cardiovascular Complications in Diabetes Postdoctoral Fellowship \#7-07-CVD-07 (A.J.A.M.). Boston University Undergraduate Research Opportunities Program (K.T.) and in part by a departmental grant from the Massachusetts Lions Organization (S.R.).

Accepted for publication March 8, 2010

Address reprint requests to Sayon Roy, Ph.D., Department of Medicine, Boston University School of Medicine, 650 Albany Street, Boston 02118 E-mail: sayon@bu.edu. 
cytes, myoblast, ventricular myocyte cells, bovine aortic endothelial cells, and mouse smooth muscle cells, exposure to $\mathrm{HG}$ has been shown to induce mitochondrial fragmentation but it is currently unknown whether mitochondrial morphology is affected by $\mathrm{HG}$ in the retinal endothelial cells and whether this impacts oxygen consumption rate, an index for mitochondrial metabolic activity. $7,8,11$

Altered mitochondrial morphology has been associated with membrane potential heterogeneity ${ }^{3}$ and increased oxidative stress in $H G$ conditions. ${ }^{7,8,11-13}$ A recent study indicated that $\mathrm{HG}$ decreases membrane potential and increases reactive oxygen species production. ${ }^{10}$ Changes in mitochondrial morphology promote the opening of the mitochondrial permeability transition pore, a critical step that leads to reduced mitochondrial membrane potential and commits cells to apoptosis. ${ }^{14}$ Interestingly, antidiabetic drug (metformin) prevented $\mathrm{HG}$-induced endothelial cell death through a mitochondrial permeability transition-dependent process. ${ }^{15}$ Other studies have implicated mitochondrial fragmentation as the precursor to mitochondrial permeability transition, which is recognized as the "point of no return" for almost all signal-transduction cascades leading to apoptosis. ${ }^{16-19}$

Although apoptosis occurs early and promotes dysfunction of the diabetic retina, the effect of $\mathrm{HG}$ on mitochondrial morphology and metabolic activity in the onset and progression of endothelial cell loss in diabetic retinopathy is unclear. In human diabetic eyes and in animal models of diabetic retinopathy, increased number of acellular capillaries and pericyte ghosts develop due to apoptotic cell loss in the retinal capillaries. ${ }^{20}$ Retinal endothelial cells and pericytes grown in HG condition show increased number of terminal deoxynucleotidyl transferase-mediated dUTP nick-end labeling (TUNEL)-positive cells, confirming that vascular cell loss seen in diabetic retinopathy is due to $\mathrm{HG}$-induced apoptosis. ${ }^{21}$ The purpose of this study was to establish if apoptosis in retinal endothelial cells involves changes in mitochondrial shape, membrane potential heterogeneity, oxygen consumption, extracellular acidification, and concomitant cytochrome $c$ release, and whether these changes are attributable to $\mathrm{HG}$ effect.

\section{Materials and Methods}

\section{Cell Culture}

RRECs were grown on poly-D-lysine-coated glass slidebottom dishes (MatTek, Ashland, MA) in Dulbecco's modified Eagle medium containing 10\% fetal bovine serum (Sigma, St. Louis), antimycotics and antibiotics. To determine the sustained effect of $\mathrm{HG}$ on mitochondrial morphology and membrane potential, cells were grown for 6 days under normal $(5 \mathrm{mmol} / \mathrm{L})$ or $\mathrm{HG}$ (30 mmol/L) medium. Following 6 days of $\mathrm{HG}$ exposure, cells were subjected to various stains and examined live using confocal microscopy. To determine time-course effect of $\mathrm{HG}$ with or without Mdivi-1 treatment (Sigma, St. Louis, MO; a selective inhibitor of mitochondrial fission) on mitochondrial morphology, cells were incubated in $\mathrm{HG}$ medium with or without Mdivi-1 (100 $\mu \mathrm{mol} / \mathrm{L})$ for $0,15,30,60$, and 120 minutes and subsequently subjected to staining and live confocal imaging.

\section{Fluorescent Probes}

To determine the sustained effect of HG on mitochondrial morphology and membrane potential heterogeneity, RRECs grown in normal or HG medium for 6 days were incubated at $37^{\circ} \mathrm{C}$ in a $5 \% \mathrm{CO}_{2}$ humidified chamber with 125 nmol/L MitoTracker Green (MTG, a membrane potential-independent dye) and $8 \mathrm{nmol} / \mathrm{L}$ tetramethylrhodamine-ethyl-ester-perchlorate (TMRE, a membrane potential-dependent dye) for 45 minutes, then washed three times with media containing $8 \mathrm{nmol} / \mathrm{L}$ TMRE and incubated with media containing $8 \mathrm{nmol} / \mathrm{L}$ TMRE for $15 \mathrm{~min}$ utes before imaging. MTG stains mitochondria green, whereas TMRE stains mitochondria red under appropriate excitation wavelength. The double staining approach facilitates proper identification of fluorescence intensity from mitochondria at different z planes. TMRE was kept in the medium while imaging. All dyes were obtained from Molecular Probes (Eugene, OR).

For time-course determination of $\mathrm{HG}$ effects on mitochondrial morphology, RRECs grown in normal medium were incubated at $37^{\circ} \mathrm{C}$ in a $5 \% \mathrm{CO}_{2}$ humidified chamber with $8 \mathrm{nmol} / \mathrm{L}$ TMRE in the normal medium for 10 minutes. TMRE was kept in the medium while imaging and cells were imaged to examine morphology of mitochondria. To examine acute effects of HG condition with or without Mdivi-1 treatment, only glucose or glucose + Mdivi-1 were added to the media to reach a final concentration of $30 \mathrm{mmol} / \mathrm{L}$ and $100 \mu \mathrm{mol} / \mathrm{L}$, respectively. Images of random fields were then captured at $0,15,30,45,60,90$, and 120 minute time points.

\section{Confocal Microscopy}

Cells were imaged live under confocal microscopy to image mitochondria using a Zeiss LSM 510 Meta microscope (Carl Zeiss, Oberkochen, Germany) with a $\times 60$ oil immersion objective. Cells were kept at $37^{\circ} \mathrm{C}$ in a $5 \% \mathrm{CO}_{2}$ humidified microscope stage chamber. MTG was subjected to $488 \mathrm{~nm}$ argon laser excitation and emission was recorded through a band pass 500 to $550 \mathrm{~nm}$ filter. TMRE was subjected to $543 \mathrm{~nm}$ helium/neon laser excitation and emission was recorded through a band-pass 650 to $710 \mathrm{~nm}$ filter. To observe individual mitochondria Z-stack images were acquired in series of 6 slices per cell ranging in thickness from 0.5 to $0.8 \mu \mathrm{m}$ per slice.

\section{Mitochondrial Morphology Analysis}

Quantitative analysis of mitochondrial morphology was conducted using computer-assisted morphometric analysis application for calculation of Form Factor (FF) and Aspect Ratio (AR) values. ${ }^{22,23}$ Acquired images of mitochondria were analyzed using $\mathrm{NIH}$-developed Image J 
software (Wayne Rasband, $\mathrm{NIH}$ ) by first processing with a median filter to obtain isolated and equalized fluorescent pixels. Mitochondria were subjected to particle analysis for acquiring FF values ( $4 \pi^{*}$ Area/perimeter $\left.{ }^{2}\right)$ and AR values derived from lengths of major and minor axes. An AR value of 1 indicates a perfect circle, and as mitochondria elongate and become more elliptical AR increases. FF value of 1 corresponds to a circular, un-branched mitochondrion, and higher FF values indicate a longer, more-branched mitochondrion.

\section{Mitochondrial Membrane Potential Heterogeneity Analysis}

To characterize mitochondrial membrane potential heterogeneity in RRECs, a ratiometric imaging approach was used by dual staining with the membrane potential-dependent dye TMRE and the membrane potential-independent dye MTG. TMRE is highly permeable across the mitochondrial membrane so equilibration is rapid, and at low concentrations TMRE does not inhibit mitochondrial respiration. The ratio product of TMRE dye to MTG dye maintains the voltage dependency of TMRE and is independent of the exact focal plane. Thus, even though the fluorescence intensity of TMRE and MTG is variable, the ratio of fluorescence intensity of TMRE to MTG dye is essentially independent of focal plane. ${ }^{13}$

Images were analyzed for membrane potential of individual mitochondrion using deviation of fluorescence intensity values for the ratio of red (TMRE) to green (MTG) dye for several mitochondria within each cell. By applying modified versions of the Nernst equation the relative membrane potential of a single mitochondrion within a cell was calculated. Within each cell the SD of all mitochondrial membrane potentials was calculated to derive the overall membrane potential heterogeneity.

\section{Cellular Oxygen Consumption and Extracellular Acidification}

Oxygen consumption and extracellular acidification rates of RRECs grown in normal or HG medium for 3 or 6 days was measured by an XF24 bioenergetic assay (Seahorse Bioscience, Billerica, MA) as previously described. ${ }^{24}$ Briefly, RRECs were plated and grown on an XF24-well microplate (Seahorse Bioscience) for 3 or 6 days in normal or HG medium to assess cellular oxygen consumption and extracellular acidification rates. Assays were initiated by removing growth medium, replacing with lowbuffered radioimmunoprecipitation assay 1640 medium containing $1 \mathrm{mmol} / \mathrm{L}$ phosphate (Molecular Devices, Sunnyvale, CA) and incubating at $37^{\circ} \mathrm{C}$ for 60 minutes to allow temperature and $\mathrm{pH}$ to reach equilibrium. The microplate was then assayed in the XF24 Extracellular Flux Analyzer (Seahorse Bioscience) to measure extracellular flux changes of oxygen and $\mathrm{pH}$ in the media immediately surrounding adherent cells. After obtaining steady state oxygen consumption and extracellular acidification rates, oligomycin (5 $\mu \mathrm{mol} / \mathrm{L})$, which inhibits ATP synthase, and proton ionophore FCCP (carbonyl cyanide 4-[trifluoromethoxy] phenylhydrazone; $1 \mu \mathrm{mol} / \mathrm{L}$ ), which uncouples mitochondria, were injected sequentially through reagent delivery chambers for each cell well in the microplate to obtain values for maximal oxygen consumption rates. Finally, a mixture containing $5 \mu \mathrm{mol} / \mathrm{L}$ rotenone (inhibitor of mitochondrial complex I) and $5 \mu \mathrm{mol} / \mathrm{L}$ myxothiazol (electron transport blocker) was injected to confirm that respiration changes were due mainly to mitochondrial respiration.

The values of oxygen consumption and extracellular acidification reflect the metabolic activities of the cells and the number of cells, so oxygen consumption and extracellular acidification rates were normalized to the total amount of cells in each well.

\section{Western Blot Analysis}

RRECs grown in normal and $H G$ were washed with PBS and lysed with $0.1 \%$ Triton-X-100 buffer containing 10 $\mathrm{mmol} / \mathrm{L}$ Tris, $\mathrm{pH} 7.5,1 \mathrm{mmol} / \mathrm{L}$ EDTA, and $1 \mathrm{mmol} / \mathrm{L}$ phenylmethylsulfonyl fluoride. The cellular extract was then centrifuged $700 \times g$ for 5 minutes. The supernatant was extracted and centrifuged again at $21000 \times g$ for 15 minutes. The supernatant was extracted as the cytosolic protein fraction. The remaining cellular pellet was washed with the same Triton buffer and centrifuged at $21000 \times g$ for 15 minutes. The supernatant was discarded and the cellular pellet was washed with radioimmunoprecipitation assay buffer containing $1 \mathrm{mmol} / \mathrm{L}$ phenylmethylsulfonyl fluoride. The washed pellet solution was centrifuged at $21000 \times g$ for 15 minutes, and the supernatant was extracted as the mitochondrial protein fraction.

An equal volume of $\times 2$ sample buffer was added to the protein samples followed by denaturation at $95^{\circ} \mathrm{C}$ for 5 minutes. Then, the protein samples were electrophoresed at $120 \mathrm{~V}$ for 50 minutes. Kaleidoscope molecular weight standards were run in separate lanes in each gel. After completion of electrophoresis, the protein samples were transferred to nitrocellulose membranes using a semidry apparatus with Towbin buffer system according to the Towbin et al procedure. ${ }^{25}$ The membranes were blocked with $5 \%$ nonfat dry milk for 1 hour and then exposed to mouse anti-cytochrome c (NeoMarkers, Fremont, CA), rabbit anti-VDAC1 (Abcam, Cambridge, MA), or rabbit anti- $\beta$-actin (Cell Signaling, Danvers, MA) in $0.2 \%$ nonfat milk overnight. After overnight incubation, the blots were washed with Tris-buffered saline containing $0.1 \%$ Tween-20 and then incubated with anti-mouse or anti-rabbit IgG secondary antibody (Sigma, St. Louis, MO) for 1 hour. The membrane was again washed as above, and then exposed to Immun-Star Chemiluminescent Protein Detection System (Bio-Rad, Temecula, CA) to detect the protein signals on an X-ray film. Densitometry was conducted and analyzed using $\mathrm{NIH} \mathrm{Image} \mathrm{anal-}$ ysis program.

\section{TUNEL Assay}

To determine apoptosis, TUNEL assay was performed on RRECs grown in normal or HG medium for 6 days using 
A
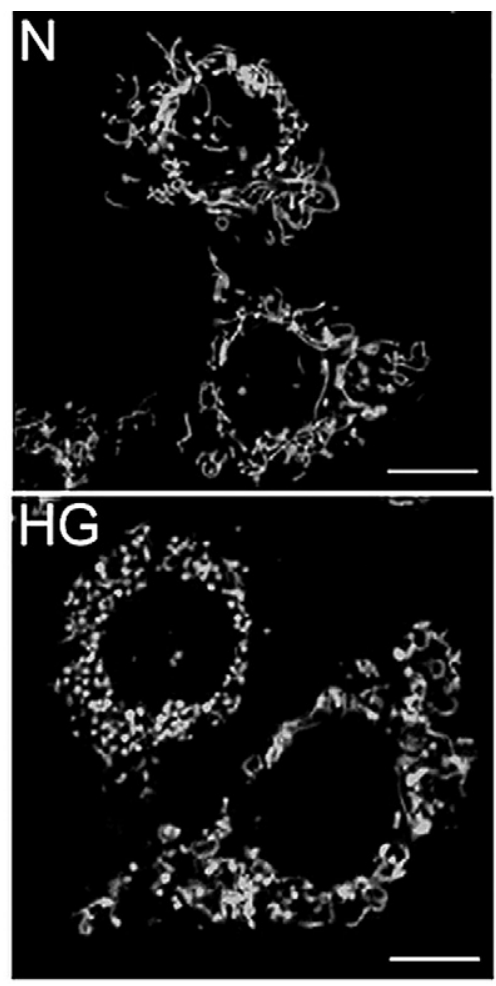

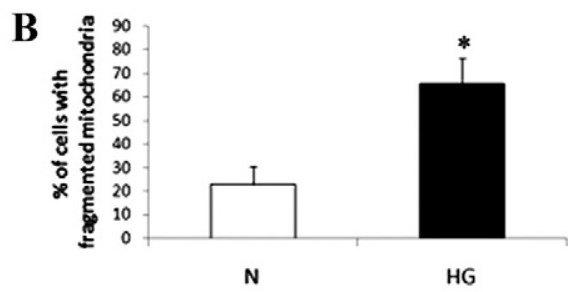

C

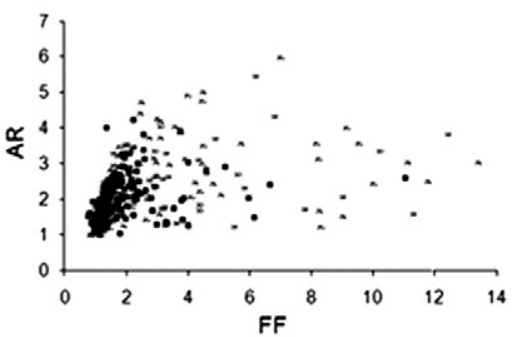

D

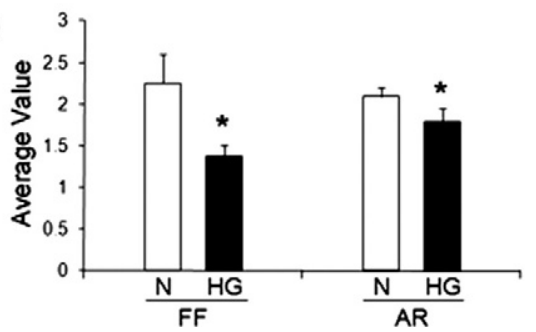

Figure 1. A-D: Mitochondrial shape change in RRECs grown in HG for six days. A: Representative confocal images of RRECs grown in normal $(\mathrm{N})$ medium and stained with TMRE showing long, tubular networks of mitochondria; RRECs grown in HG medium for six days show significant mitochondrial fragmentation. Scale bar $=20 \mu \mathrm{m}$. B: Of the total cell population, a significant percentage of RRECs grown in HG for six days have fragmented mitochondria, compared with RRECs grown in normal medium. C: The graph shows FF versus AR values for individual mitochondrion within RRECs grown in normal or HG medium for six days. Mitochondria of RRECs grown in normal medium have greater $\mathrm{FF}$ and $\mathrm{AR}$ values corresponding to a long, tubular shape. Under HG condition, mitochondria have lower FF and AR values indicating fragmentation and punctuate shape. Crosses = normal, black squares = HG. D: The graph shows average FF and AR values for mitochondria of RRECs grown in normal or HG medium for six days. Mitochondria were significantly fragmented in RRECs grown in HG medium, as shown by the decreased $\mathrm{FF}$ and AR values. ${ }^{*} P<$ $0.01, n=6$. the ApopTag In Situ Apoptosis Detection kit (Chemicon, Temecula, CA) according to the manufacturer's instruction. Briefly, cells grown on coverslips were fixed with $4 \%$ paraformaldehyde, then permeated with pre-cooled mixture of 2:1 ratio of ethanol to acetic acid. After two washes in PBS, cells were incubated with equilibration buffer and then incubated with TdT enzyme in a moist chamber at $37^{\circ} \mathrm{C}$ for 1 hour. Cells were then washed with PBS and incubated with anti-digoxigenin peroxidase. Finally, the cells were washed in PBS and mounted in SlowFade (Molecular Probes, Eugene, OR). Images from ten random fields representing each coverslip were captured using a digital Nikon DS-Fi1 microscope and recorded for analysis.

\section{Statistics}

All data were expressed as mean $\pm \mathrm{SD}$. Comparisons between groups were performed with Student's $t$-test. A value of $P<0.05$ was considered statistically significant.

\section{Results}

\section{HG Exposure of Six Days Induces Mitochondrial Morphology Change in RRECs}

Mitochondria exist as long, tubular networks throughout the cytoplasm in RRECs grown in normal medium. RRECs grown in HG medium for 6 days exhibited significant mitochondrial fragmentation appearing round with punctuate morphology (Figure 1A). Of the total cell populations observed, a significant percentage of RRECs grown in $\mathrm{HG}$ exhibited punctuate mitochondria compared with normal cells (Figure 1B; $65.5 \pm 10.9 \%$ of cells in $H G$ vs. $23.0 \pm 7.7 \%$ in normal, $P<0.001, n=8$ ). Plotting AR versus FF indicates that the majority of mitochondria from RRECs grown in $H G$ media had lower values of FF and AR than RRECs grown in normal medium (Figure 1C). Accordingly, RRECs grown in HG medium display lower average values of FF and AR than RRECs grown in normal medium, indicating significant fragmentation of mitochondria due to $\mathrm{HG}$ condition (Figure 1D; FF for HG: 1.377 compared with 2.248 in normal, $P<0.001$, AR for HG: 1.793 compared with 2.094 in normal, $P=0.0055, n=8$ ).

\section{Mdivi-1 Prevents Acute HG-induced Mitochondrial Fragmentation in RRECs}

Acute exposure of RRECs to $H G$ medium resulted in transient fragmentation of mitochondria within 30 minutes, followed by partial morphology recovery after 60 minutes (Figure 2A; 0 minutes. $H G$ : $F F=2.38, A R=2.11$; 30 minutes. $\mathrm{HG}$ : $F F=1.519 P=0.0003, A R=1.89 P=$ $0.009 ; 120$ minutes. $\mathrm{HG}$ : $\mathrm{FF}=2.01 P=0.0006, \mathrm{AR}=$ $2.06 P=0.004, n=6$ ). The mitochondrial morphology recovered within an hour resembling the normal mitochondrial network. However, at the 48-hour time point we observed fragmentation again that remained for the rest of the 6-day experiment. These findings indicate that mitochondrial fragmentation in the presence of $\mathrm{HG}$ exhibits a bimodal morphological change: a rapid, transient fragmentation followed by a recovery phase, and then 
A

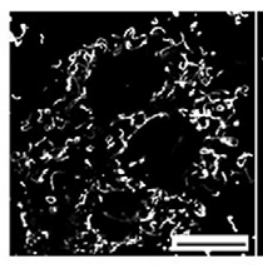

$0 \mathrm{~min}$.

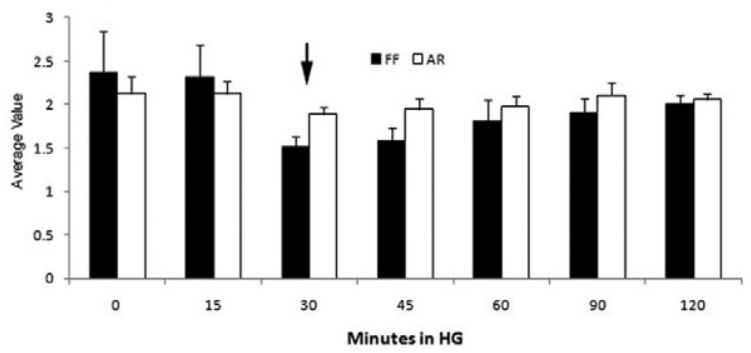

B

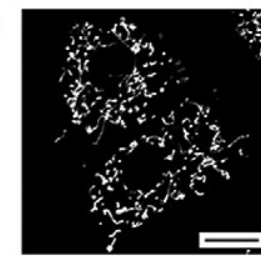

0 min.

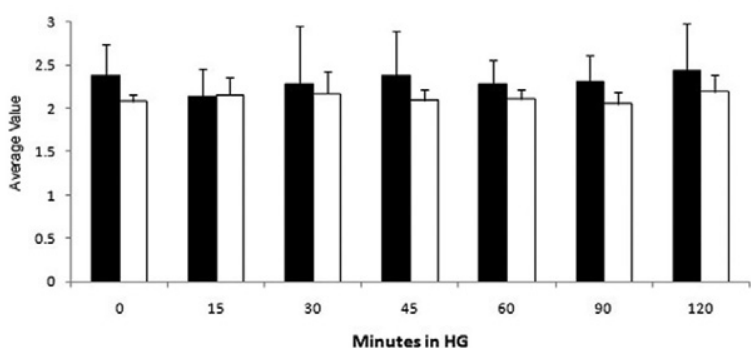

Figure 2. A-B: Transient change in mitochondrial morphology after acute HG exposure is preventable with Mdivi-1 in RRECs. A: RRECs stained with TMRE and exposed to HG exhibit significant mitochondrial fragmentation at 30 minutes, followed by morphology recovery after 90 minutes. The graph shows average $\mathrm{FF}$ and $\mathrm{AR}$ values for mitochondria of RRECs grown in HG medium at $0,15,30,45,60,90$, and 120 minutes. Within 30 minutes of HG incubation mitochondria significantly fragmented with $\mathrm{FF}$ and AR values decreasing to similar values seen at six days of HG incubation (arrow indicates the onset of significant fragmentation, $P<0.01, n=6$ ). After 60 minutes of HG incubation, substantial morphology recovery was observed as indicated by the increase in FF and AR values. This morphology recovery continued through the 120 minute incubation period, indicating that RRECs can transiently recover from acute HG insult. B: RRECs treated with mitochondrial fission inhibitor Mdivi-1 and exposed to HG for 30 or 90 minutes show no significant morphological change. Accordingly, FF and AR values of these mitochondria do not vary significantly over the 120-minute exposure to HG. This may implicate mitochondrial fission in the transient fragmentation of RREC mitochondria in response to acute HG exposure. Scale bar $=20 \mu \mathrm{m}$.

with sustained exposure to $\mathrm{HG}$ undergoes permanent mitochondrial fragmentation.

To determine whether transient mitochondrial fragmentation by acute $H G$ exposure is related to increased fission, Mdivi-1, a selective inhibitor of mitochondrial division and fission proteins dynamin-related GTPase and dynamin, was used to inhibit fission events that appeared with acute HG exposure. Effects of Mdivi-1 on mitochondrial morphology or dysfunction with prolonged HG exposure could not be assessed as treatment of $>4$ hours with Mdivi-1 causes cell death. ${ }^{26}$ Cells treated with 100 $\mu \mathrm{mol} / \mathrm{L}$ Mdivi-1 simultaneously with $\mathrm{HG}$ exposure did not exhibit significant morphology change compared with untreated cells exposed to HG (Figure 2B; $n=6$ ). Thus, transient mitochondrial fragmentation due to acute $\mathrm{HG}$ exposure may be mediated by increased mitochondrial fission, and is preventable on treatment with Mdivi-1.

\section{HG Increases Membrane Potential Heterogeneity in Mitochondria of RRECs}

RRECs grown in HG medium for 6 days and doublestained with TMRE and MTG dyes showed greater variation in mitochondrial membrane potential within a cell, as seen by greater range of green (depolarized) to red (hyperpolarized) color compared with those grown in normal medium (Figure 3A). By measuring the fluorescence intensity deviation of several mitochondria within a single cell, mitochondrial membrane potential heterogeneity could be quantified (Figure 3B; fluorescence intensity deviation for HG: 354.5 compared with 182.9 in normal, $P=0.0001, n=8)$. Therefore, $H G$ conditions not only disrupt mitochondrial morphology by fragmenting the mitochondrial network, but also the membrane potentials of the mitochondria are disrupted resulting in different mitochondria within a cell exhibiting different membrane potentials that are more heterogenic than those of normal control cells.

\section{HG-Induced Mitochondrial Morphology and Membrane Potential Changes are Concomitant with Decreased Steady State/Maximal Oxygen Consumption and Increased Extracellular Acidification in RRECs}

To assess HG-induced changes in RREC metabolic capacity and extracellular acidification, RRECs grown for 3 or 6 days in normal or $\mathrm{HG}$ conditions were measured simultaneously for rates of cellular oxygen consumption and extracellular acidification using XF24 bioenergetic assay. The experimental design and assessment are depicted in the line graph of Figure 4A. Steady-state oxygen consumption and extracellular acidification were measured at the fourth time point (Figure 4A). Subsequently, oligomycin (injection vertical line A; Figure 4A) was injected to inhibit ATP synthase, followed by addition of FCCP (injection vertical line B; Figure 4A) to uncouple mitochondria and obtain values for maximal oxygen consumption at the tenth time point. Finally, rotenone and myxothiazol were injected (injection vertical line C; Figure $4 \mathrm{~A})$ to confirm that the respiration changes were due mainly to mitochondrial respiration.

After 3 days of $H G$ exposure RRECs showed no significant change in steady state or maximal oxygen consumption $(n=6)$. Prolonged HG exposure of 6 days resulted in significant decrease of steady state and maximal oxygen consumption (Figure 4A; steady state: 1.55 vs. $1.99 \mathrm{nmol} \mathrm{O}_{2} / \mathrm{min} / 10^{6}$ cells in normal, $P=0.016$; maximal: 1.89 vs. $3.10 \mathrm{nmol} \mathrm{O}_{2} / \mathrm{min} / 10^{6}$ cells in normal, $P=$ $0.014, n=6)$. 

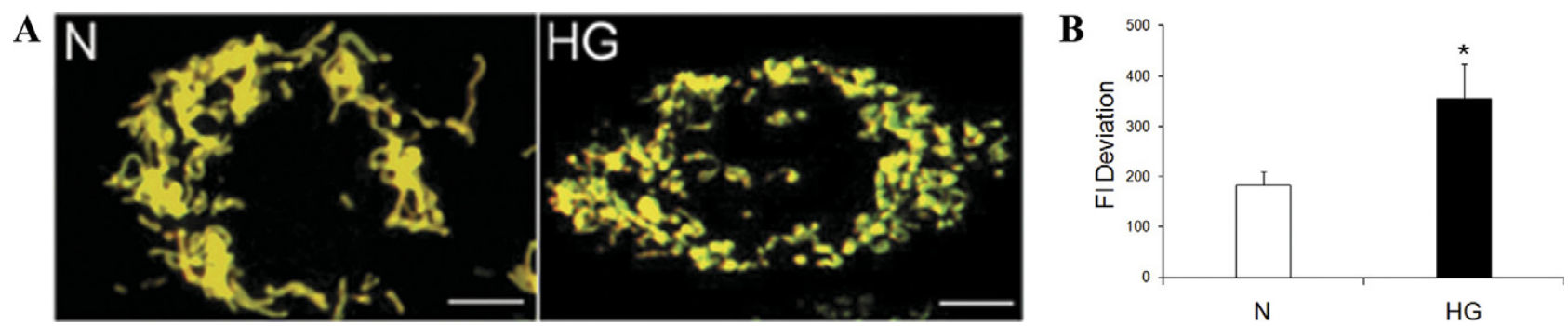

Figure 3. A-B: Increase in mitochondrial membrane potential heterogeneity of RRECs grown in HG for six days. A: RRECs grown for six days in normal (N) or HG medium were double-stained with mitochondrial membrane potential-sensitive TMRE (red) dye and membrane potential-independent MTG (green) dye. Mitochondria of RRECs grown in normal medium are uniformly yellow, indicating little variation in membrane potential among the mitochondrial population, whereas mitochondria of RRECs grown in HG medium vary in color (yellow, green, and orange), indicating varied uptake of TMRE and heterogeneic membrane potential within the mitochondrial network. Scale bar $=10 \mu \mathrm{m}$. B: The graph shows the SD of the fluorescence intensity membrane potentials for several mitochondria within RRECs grown in normal or HG media for six days. RRECs grown in HG had a greater SD of the membrane potentials indicating significant increase in the heterogeneity of mitochondrial membrane potential. ${ }^{*} P<0.05, n=6$.

To determine whether RRECs can compensate for reduced metabolic capacity (oxidative phosphorylation) of the mitochondria in $\mathrm{HG}$ conditions, we examined if extracellular acidification rates were simultaneously increasing for RRECs grown for 3 or 6 days of HG; such changes would indicate increased rate of glycolysis in the cells. RRECs grown for 3 days in HG showed no change in extracellular acidification but significant increase was observed after 6 days of HG exposure (Figure 4B; $135 \pm$ $12 \%$ of control, $P=0.005, n=6)$. The results may indicate that RRECs increase glycolysis to compensate for HG-induced decreases in metabolic capacity, as displayed by decreased oxygen consumption.

Oxygen consumption may be influenced by the number of mitochondria; thus to assess mitochondrial content of RRECs grown in normal or HG medium for 6 days, whole cell extract was assessed for VDAC1 expression by Western blot. HG exposure of 6 days did not alter VDAC1 expression (Figure $4 C ; n=6$ ), suggesting no change in mitochondrial content in cells grown in HG for 6 days. Taken together, the data suggests that sustained exposure of RRECs to HG conditions results in significant mitochondrial fragmentation and subsequent decrease in mitochondrial metabolic capacity, as indicated by decreased maximal oxygen consumption.

\section{Increased Cytochrome c Release and Number of TUNEL-Positive Cells Occurs with HG- Induced Mitochondrial Morphology and Functional Changes}

RRECs grown for 6 days in normal or HG conditions were harvested for mitochondrial and cytosolic protein by differential centrifugation. Cells exposed to HG for 6 days showed a significant increase in cytochrome c protein levels in the cytosol as determined by Western blot anal-
A
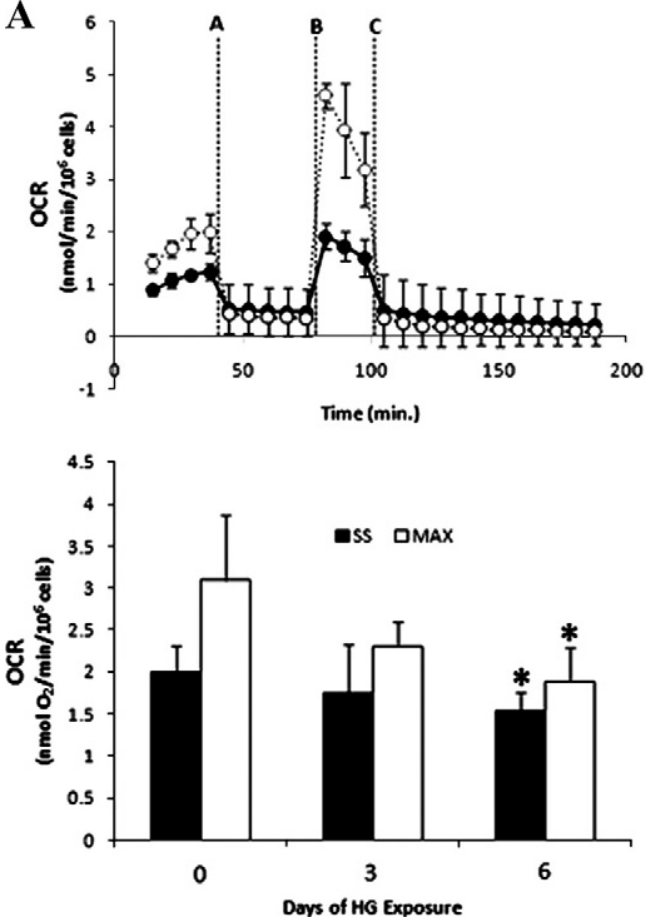

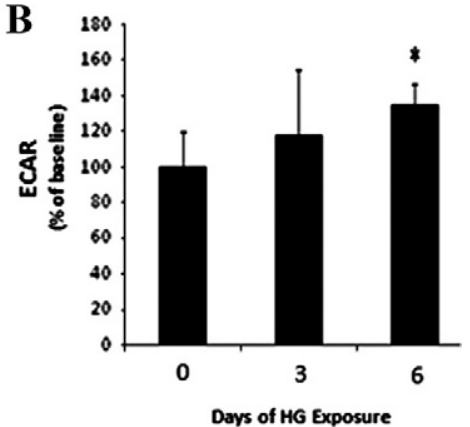

C

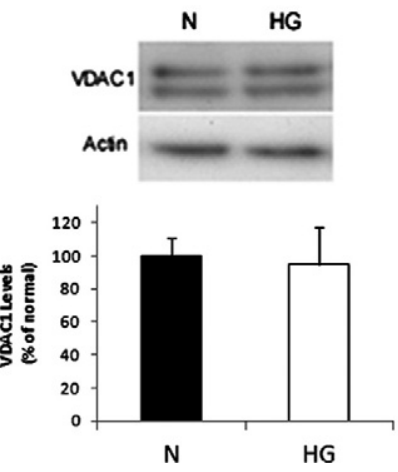

Figure 4. A-C: Changes in oxygen consumption and extracellular acidification of RRECs grown in HG medium. A: The line graph shows the experimental design and assessment of steady-state and maximal oxygen consumption (OCR) for RRECs grown in normal or HG for six days (White circles/dashed line is normal, Black circles/dark line is HG). Dotted-lines A, B, and C indicate injections of oligomycin, FCCP, and rotenone/myxothiazol, respectively. Steady-state oxygen consumption was measured at the fourth time point before oligomycin injection, while maximal oxygen consumption was measured at the tenth time point after FCCP injection. The bar graph shows that sustained HG exposure of six days resulted in significant decreases in steady state and maximal oxygen consumption rates. B: Extracellular acidification (ECAR) was measured at the fourth time point and was significantly increased after six days of HG exposure compared with normal. C: VDAC1 protein levels of RRECs grown in normal $(\mathrm{N})$ or HG medium for six days. No change in VDAC1 expression was observed after six days of $\mathrm{HG}$, indicating that total mitochondrial content is not affected in RRECs grown in HG for six days. ${ }^{*} P<$ $0.05, n=6$. 
A

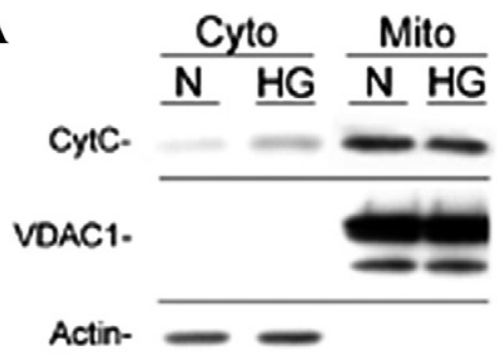

B

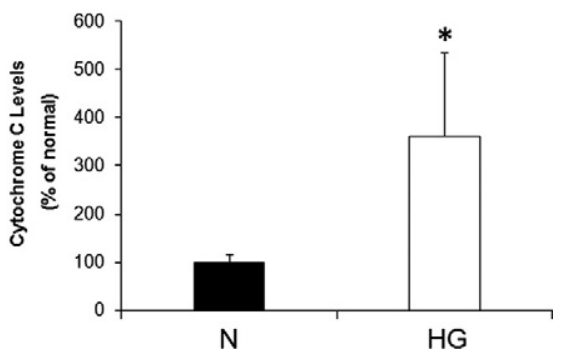

Figure 5. A-B: HG exposure of six days increases cytochrome c release into the cytosol of RRECs. A: Cytosolic and mitochondrial protein fractions were isolated from RRECs grown in normal $(\mathrm{N})$ or HG medium for six days and cytochrome c release in the cytosol was assessed by Western blot analysis. Purity/loading of the mitochondrial fraction was confirmed by VDAC1 staining, and purity/loading of the cytosolic fraction was confirmed by actin staining. B: RRECs grown in HG medium for six days showed increased level of cytochrome $\mathrm{c}$ in the cytosol. ${ }^{*} P<0.05, n=6$ ysis (Figure $5 \mathrm{~A}-\mathrm{B} ; 361 \pm 174 \%$ of control, $P=0.015, n=$ 6). Thus, the HG-induced shape and functional changes of mitochondria coincided with increased release of cytochrome c from the mitochondria into the cytosol. This may indicate that the observed effects of $H G$ on mitochondrial morphology, membrane potential, and respiration may facilitate the release of cytochrome $\mathrm{c}$ from mitochondria and the progression to apoptosis. Furthermore, to confirm that $\mathrm{HG}$-induced cytochrome c release occurs during accelerated apoptosis in retinal endothelial cells, TUNEL-staining was performed on RRECs grown for 6 days in normal or HG, when cytochrome $\mathrm{c}$ release was observed. The number of TUNEL-positive cells was significantly increased in RRECs grown for 6 days in $\mathrm{HG}$ compared with those grown in normal medium (Figure 6A-B, $223 \pm 57 \%$ of normal, $P=$ $0.042, n=6)$.

\section{Discussion}

In this study, we have examined whether HG alters mitochondrial shape and whether the changes in mitochondrial shape play a role in inducing apoptosis. Additionally, we investigated whether mitochondrial shape change alters mitochondrial metabolic activity by examining steady-state and maximal oxygen consumption and mitochondrial membrane potential heterogeneity. Our results indicate that HG induces mitochondrial fragmentation, mitochondrial metabolic activity with concomitant cytochrome c release under HG condition. Interestingly our data shows that $\mathrm{HG}$ induces mitochondrial fragmentation in two phases: a rapid, initial change that occurs in one hour from which the fragmented mitochondria recovers within the next couple of hours; and a sustained change that occurs after two additional days in $\mathrm{HG}$ when permanent fragmentation occurs. The implications of this bimodal display of mitochondrial fragmentation under HG condi- tion remain unclear. A transient increase in reactive oxygen species level mediated by HG was previously reported to match the transient temporal profile of mitochondrial shape change in rat myoblasts. ${ }^{8}$

Disruption in mitochondrial functioning is an early signature of apoptosis. ${ }^{27}$ Studies have shown that hyperglycemia induces mitochondrial dysfunction but it is unknown how mitochondrial morphology change may exacerbate other pathways. For example, mitochondrial dysfunction is associated with increased oxidative stress in diabetic retinopathy, which can affect pro- and antiapoptotic gene expression, signal transduction, and mitochondrial membrane potential, triggering apoptosis. ${ }^{12}$ A study has shown that mitochondrial dysfunction may be associated with increased Bax recruitment and expression, and cytochrome c release, implicating its involvement in the acceleration of retinal vascular cells dysfunction seen in diabetes. ${ }^{28}$ A recent finding highlighted how reactive oxygen species overproduction due to $H G$ can increase damage to mitochondrial DNA, which can then further disrupt mitochondrial membrane potential and impair functioning of mitochondria. ${ }^{10}$

Though these studies have reported on the effects of $\mathrm{HG}$-induced oxidative stress on mitochondrial functioning, it is still unknown how HG conditions affect the morphology of mitochondria and how morphology changes may influence mitochondrial functioning and metabolic activity, culminating in apoptosis. Our work for the first time demonstrates how HG fragments mitochondria in retinal endothelial cells. This morphology change may lead to a functional collapse of the mitochondrial population, as shown by significant membrane potential heterogeneity and decreased oxygen consumption. Increased glycolysis may help compensate the HG-induced decrease in mitochondria metabolism. Furthermore, the mitochondrial shape change and dysfunction was concomitant with increased cytochrome $\mathrm{c}$ release from the mitochondria into
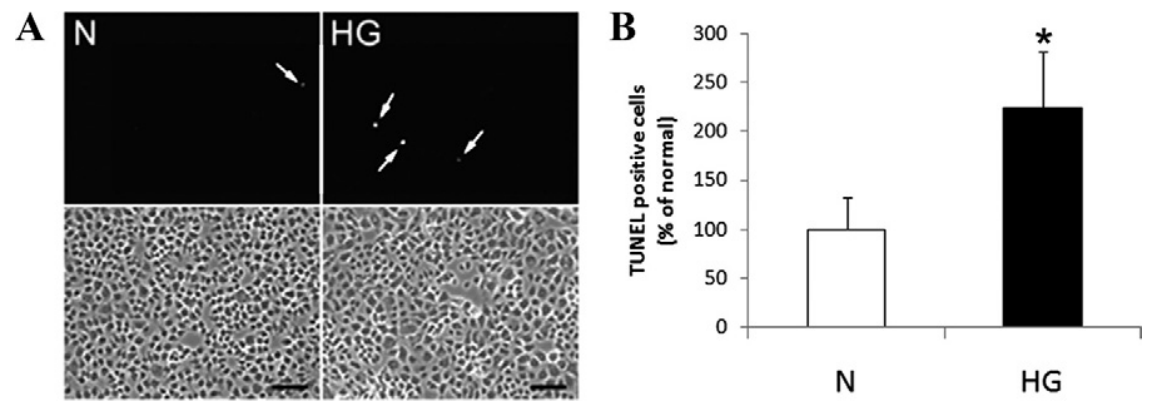

Figure 6. A-B: Accelerated apoptosis of RRECs grown in HG for six days. A: TUNEL-positive cells in RRECs grown in normal (N) or HG medium for six days. The number of TUNEL-positive cells (indicated by arrows) was significantly increased in RRECs grown in HG compared with those of cells grown in normal medium. Scale bar $=50 \mu \mathrm{m}$. B: The graph indicates that RRECs grown in HG for six days show a significant increase in the number of TUNEL-positive cells compared with those of normal cells. ${ }^{*} P<0.05$ $n=6$. 
the cytosol. Overall, the findings indicate a new step in the pathway for accelerated apoptosis in retinal endothelial cells, including a direct effect of $\mathrm{HG}$ on mitochondrial morphology, which when compromised leads to further mitochondrial dysfunction, cytochrome c release, and apoptosis.

Heterogeneity in mitochondrial membrane potential has been observed in numerous cell types and is thought to reflect varying states of mitochondrial function. These differences in membrane potential may be due to uneven distribution of respiratory chain components, uneven access to metabolites and calcium, or changes in mitochondrial localization. ${ }^{29}$ Increased levels of heterogeneity have been associated with increased cellular stress. For example, studies investigating the ischemia-reperfusion model has shown that rat cardiomyocytes exhibit higher levels of heterogeneity after reperfusion. ${ }^{30,31}$ Moreover, increased nutrient levels have been shown to increase mitochondrial membrane potential heterogeneity in pancreatic $\beta$ cells. ${ }^{13}$ We report that an increase in mitochondrial heterogeneity coincides with the progression of RRECs through apoptosis. In other systems, it has been reported that increased mitochondrial heterogeneity may reflect permeability transition pore opening and the release of cytochrome c. ${ }^{32,33}$

Our findings coincide with recent studies of mitochondria biology that have focused on the role of mitochondrial morphology as a regulator of mitochondrial functioning. Most mammalian cell types have long, tubular networks of mitochondria, which is critical to normal mitochondria functioning. ${ }^{34,35}$ These elongated networks of mitochondria are regulated by fusion and fission events that involve the formation or breaking of the mitochondria network, respectively. If either fusion is decreased or fission increased, fragmentation of the mitochondrial network can occur, resulting in shorter, punctuate mitochondria. ${ }^{5}$ Notably, this fragmentation is associated with mitochondrial dysfunction, leading to increased production of reactive oxygen species. HG-induced mitochondrial fragmentation may thus play a significant role in the development of diabetic retinopathy.

\section{Acknowledgment}

We thank Seahorse Bioscience for their help and use of equipment.

\section{References}

1. Antonetti DA, Lieth E, Barber AJ, Gardner TW: Molecular mechanisms of vascular permeability in diabetic retinopathy. Semin Ophthalmol 1999, 14:240-248

2. Jeong SY, Seol DW: The role of mitochondria in apoptosis. BMB Rep 2008, 41:11-22

3. Chen H, Chomyn A, Chan DC: Disruption of fusion results in mitochondrial heterogeneity and dysfunction. J Biol Chem 2005, 280: 26185-26192

4. Ishihara N, Jofuku A, Eura Y, Mihara K: Regulation of mitochondrial morphology by membrane potential, and DRP1-dependent division and FZO1-dependent fusion reaction in mammalian cells. Biochem Biophys Res Commun 2003, 301:891-898
5. Scorrano L: Multiple functions of mitochondria-shaping proteins. Novartis Found Symp 2007, 287:47-55; discussion 55-49

6. Perfettini JL, Roumier T, Kroemer G: Mitochondrial fusion and fission in the control of apoptosis. Trends Cell Biol 2005, 15:179-183

7. Yu T, Sheu SS, Robotham JL, Yoon Y: Mitochondrial fission mediates high glucose-induced cell death through elevated production of reactive oxygen species. Cardiovasc Res 2008, 79:341-351

8. Yu T, Robotham JL, Yoon Y: Increased production of reactive oxygen species in hyperglycemic conditions requires dynamic change of mitochondrial morphology. Proc Natl Acad Sci USA 2006, 103: 2653-2658

9. Li AF, Roy S: High glucose-induced downregulation of connexin 43 expression promotes apoptosis in microvascular endothelial cells. Invest Ophthalmol Vis Sci 2009, 50:1400-1407

10. Xie L, Zhu X, Hu Y, Li T, Gao Y, Shi Y, Tang S: Mitochondrial DNA oxidative damage triggering mitochondrial dysfunction and apoptosis in high glucose-induced HRECs. Invest Ophthalmol Vis Sci 2008, 49:4203-4209

11. Paltauf-Doburzynska J, Malli R, Graier WF: Hyperglycemic conditions affect shape and $\mathrm{Ca} 2+$ homeostasis of mitochondria in endothelial cells. J Cardiovasc Pharmacol 2004, 44:423-436

12. Kowluru RA: Diabetic retinopathy: mitochondrial dysfunction and retinal capillary cell death. Antioxid Redox Signal 2005, 7:1581-1587

13. Wikstrom JD, Katzman SM, Mohamed H, Twig G, Graf SA, Heart E, Molina AJ, Corkey BE, de Vargas LM, Danial NN, Collins S, Shirihai OS: beta-Cell mitochondria exhibit membrane potential heterogeneity that can be altered by stimulatory or toxic fuel levels. Diabetes 2007, $56: 2569-2578$

14. Wasilewski M, Scorrano L: The changing shape of mitochondrial apoptosis. Trends Endocrinol Metab 2009, 20:287-294

15. Detaille D, Guigas B, Chauvin C, Batandier C, Fontaine E, Wiernsperger N, Leverve $X$ : Metformin prevents high-glucose-induced endothelial cell death through a mitochondrial permeability transition-dependent process. Diabetes 2005, 54:2179-2187

16. Frank S, Gaume B, Bergmann-Leitner ES, Leitner WW, Robert EG, Catez F, Smith CL, Youle RJ: The role of dynamin-related protein 1, a mediator of mitochondrial fission, in apoptosis. Dev Cell 2001, 1:515-525

17. Breckenridge DG, Stojanovic M, Marcellus RC, Shore GC: Caspase cleavage product of BAP31 induces mitochondrial fission through endoplasmic reticulum calcium signals, enhancing cytochrome c release to the cytosol. J Cell Biol 2003, 160:1115-1127

18. Hwang JU, Gu Y, Lee YJ, Yang Z: Oscillatory ROP GTPase activation leads the oscillatory polarized growth of pollen tubes. Mol Biol Cell 2005, 16:5385-5399

19. Sugioka R, Shimizu S, Tsujimoto Y: Fzo1, a protein involved in mitochondrial fusion, inhibits apoptosis. J Biol Chem 2004, 279:5272652734

20. Kern TS, Engerman RL: A mouse model of diabetic retinopathy. Arch Ophthalmol 1996, 114:986-990

21. Garcia M, Vecino E: [Intracellular pathways leading to apoptosis of retinal cells]. Arch Soc Esp Oftalmol 2003, 78:351-364

22. De Vos KJ, Allan VJ, Grierson AJ, Sheetz MP: Mitochondrial function and actin regulate dynamin-related protein 1-dependent mitochondrial fission. Curr Biol 2005, 15:678-683

23. Koopman WJ, Verkaart S, Visch HJ, van der Westhuizen FH, Murphy MP, van den Heuvel LW, Smeitink JA, Willems PH: Inhibition of complex I of the electron transport chain causes O2-mediated mitochondrial outgrowth. Am J Physiol Cell Physiol 2005, 288:C1440C1450

24. Wu M, Neilson A, Swift AL, Moran R, Tamagnine J, Parslow D, Armistead S, Lemire K, Orrell J, Teich J, Chomicz S, Ferrick DA: Multiparameter metabolic analysis reveals a close link between attenuated mitochondrial bioenergetic function and enhanced glycolysis dependency in human tumor cells. Am J Physiol Cell Physiol 2007, 292:C125-C136

25. Towbin H, Staehelin T, Gordon J: Electrophoretic transfer of proteins from polyacrylamide gels to nitrocellulose sheets: procedure and some applications. Proc Natl Acad Sci USA 1979, 76:4350-4354

26. Mitra K, Wunder C, Roysam B, Lin G, Lippincott-Schwartz J: A hyperfused mitochondrial state achieved at G1-S regulates cyclin E buildup and entry into S phase. Proc Natl Acad Sci USA 2009, 106:11960-11965

27. Rego AC, Oliveira CR: Mitochondrial dysfunction and reactive oxygen 
species in excitotoxicity and apoptosis: implications for the pathogenesis of neurodegenerative diseases. Neurochem Res 2003, 28:1563-1574

28. Podesta F, Romeo G, Liu WH, Krajewski S, Reed JC, Gerhardinger C Lorenzi M: Bax is increased in the retina of diabetic subjects and is associated with pericyte apoptosis in vivo and in vitro. Am J Pathol 2000, 156:1025-1032

29. Wikstrom JD, Twig G, Shirihai OS: What can mitochondrial heterogeneity tell us about mitochondrial dynamics and autophagy? Int J Biochem Cell Biol 2009, 41:1914-1927

30. Kuznetsov AV, Schneeberger S, Renz O, Meusburger H, Saks V, Usson Y, Margreiter R: Functional heterogeneity of mitochondria after cardiac cold ischemia and reperfusion revealed by confocal imaging. Transplantation 2004, 77:754-756

31. Kuznetsov AV, Usson Y, Leverve X, Margreiter R: Subcellular heteroge- neity of mitochondrial function and dysfunction: evidence obtained by confocal imaging. Mol Cell Biochem 2004, 256-257:359-365

32. D'Herde K, De Prest B, Mussche S, Schotte P, Beyaert R, Coster RV Roels F: Ultrastructural localization of cytochrome $\mathrm{C}$ in apoptosis demonstrates mitochondrial heterogeneity. Cell Death Differ 2000, 7:331-337

33. Krysko DV, Roels F, Leybaert L, D'Herde K: Mitochondrial transmembrane potential changes support the concept of mitochondrial heterogeneity during apoptosis. J Histochem Cytochem 2001, 49:12771284

34. Karbowski M, Youle RJ: Dynamics of mitochondrial morphology in healthy cells and during apoptosis. Cell Death Differ 2003, 10:870880

35. Chen $\mathrm{H}$, Chan DC: Emerging functions of mammalian mitochondria fusion and fission. Hum Mol Genet 2005, 14 Spec No. 2:R283-R289 